\title{
EXPERIENCIAS
}

\section{Acercamiento a la realidad de las familias de personas con autismo}

\section{Approaching the children' families with autism}

\section{M. áNGELES MARTÍNEZ MARTÍN* M. ${ }^{\text {a }}$ CRUZ BILBAO LEÓN*}

\begin{abstract}
RESUMEN
Es un hecho universalmente aceptado que la presencia en las familias de un hijo con algún tipo de minusvalia fisica o psíquica grave constituye un factor potencial que perturba la dinámica familiar. Esto es porque la convivencia con una persona con este tipo de problemas puede provocar cambios importantes en la vida personal de los distintos miembros -fundamentalmente los padres- y de las relaciones entre ellos.

Los datos muestran de manera indiscutible que muchas familias con hijos con autismo presentan niveles de estrés crónico, significativamente superiores a los que presentan las familias con hijos con otras discapacidades. Esta diferenciación en cuanto al efecto causado por el autismo en la familia, se inicia muchas veces a causa de las incertidumbres que plantea la evolución disarmónica de los niños y niñas con autismo, se mantiene posteriormente ante la ausencia de un diagnóstico y pronóstico claros y aumenta considerablemente como consecuencia de los múltiples efectos negativos en el ámbito familiar. La convivencia en el hogar de un niño o niña con autismo suele ser muy compleja y las familias se ven sometidas desde el principio a modificaciones severas de su régimen de vida habitual con limitaciones desmedidas de su independencia.
\end{abstract}

\section{PALABRAS CLAVE}

Trastornos del espectro autista, Duelo, Impacto, Afrontamiento, Estrés familiar.

* Área de Personalidad, Evaluación y Tratamiento Psicológico. Facultad de Humanidades y Educación. Universidad de Burgos. 
Acercamiento a la realidad de las familias de personas con autismo

\section{ABSTRACT}

It is a universally accepted fact that the presence in the families of a child with some type of physical or psychic serious handicap constitutes a potential factor that disturbs the familiar dynamics. This is because the cohabitation with a person with this type of problems can provoke important changes in the personal life of the different members - fundamentally the parents - and of the relations between them.

The information shows that many children families of with autism present levels of stress, significantly higher to whom present the families with children with other disabilities. This differentiation on the effect caused by autism in the family begins often due to the uncertainties on the not harmonic evolution of the children with autism, and is kept later by the absence of a diagnosis and increases considerably as consequence of the multiple negative effects in the familiar area. The coexistence at home with a child with autism is in the habit of being very complex and families are submitted from the beginning to severe modifications of their habitual life with excessive limitations of their independence.

\section{KEY WORDS}

Disorders of the autistic spectrum, Duel, Impact, Confrontation, Familiar stress. 


\section{TOMA DE CONCIENCIA}

Tener un hijo es uno de los acontecimientos vitales más importantes para un ser humano. Los vínculos afectivos entre padres e hijos, así como las emociones que se ponen en juego, son normalmente intensos. Una hija o un hijo es siempre fuente de ilusiones y miedos. El hecho de que ser padres sea algo habitual no significa que sea fácil, y cuando el hijo tiene alguna dificultad grave todo puede resultar especialmente difícil (Paniagua, 1999).

Normalmente, los padres de un niño/a con trastorno del espectro autista - en adelante TEA- disfrutan durante un tiempo de la enorme felicidad que supone el tener un hijo, un niño que todo el mundo considera que se desarrolla como cualquier otro. Sin embargo, en un momento del desarrollo, normalmente a partir del primer año, empiezan a aparecer ciertos comportamientos que desconciertan a los padres, sintomas que les provocan una inquietud que aumenta con el paso del tiempo. El rechazo al contacto afectivo, la falta de respuesta a las instrucciones verbales, el juego repetitivo, son aspectos que impactan profundamente. En muchas ocasiones ante estas señales los padres buscan respuestas en los profesionales, especialmente en los pediatras, respuestas que no encuentran.

El hecho de que la mayoría de los niños con autismo no presentan alteraciones fisicas evidentes dificulta la toma de conciencia del problema. Frecuentemente el autismo frustra la realización primera de las intensas emociones de crianza que se derivan de la preparación biológica para la reproducción (Rivière, 1997a).

"La vida de las personas con autismo es muy dura porque su discapaci- dad no se manifiesta a primera vista $y$ es dificil de entender" (Stanton, 2002, p.17).

El presentar rasgos físicos normales, unido al hecho de que en muchos casos manifiestan niveles aceptables en algunas áreas evolutivas, genera enormes dudas a los padres, llegando a creer que su hijo puede presentar algún problema pero no un trastorno grave e irreversible como es el autismo. Si a esto añadimos el que los propios profesionales suelen responder ante las inquietudes de los padres, tranquilizándoles y asegurándoles que su hijo no tiene ningún problema, y que intenten comportarse como padres normales, y no analizar tanto la evolución de su hijo (Cuxart, 1997), podemos entender la enorme incertidumbre por la que pasan estas familias. Esperanza y desesperanza conviven generando un efecto doloroso en quienes observan un niño de apariencia normal, con un desarrollo también normal en el primer año pero con una serie de pautas extrañas de conducta, con una especie de soledad (Rivière, 1997b), de indiferencia hacia las personas.

La toma de conciencia en el caso del autismo es especialmente difícil puesto que se trata de un trastorno asociado a una cierta ambigüedad debido, entre otros aspectos, a la amplia variabilidad del pronóstico, a la ausencia de marcadores biológicos, a la dificultad de los padres para detectar sintomas tan sutiles como los que caracterizan al autismo en sus primeras etapas, a la gran variabilidad que presenta. El autismo no es un trastorno uniforme, ni absolutamente demarcado, y su presentación oscila en un espectro de mayor a menor afectación; varía con el tiempo, y se ve influido por factores como el grado de capacidad intelectual asociada o el acceso a apoyos especializados. 
Acercamiento a la realidad de las familias de personas con autismo

Es muy posible que en el autismo y otros trastornos del espectro autista, los primeros signos pasen inadvertidos aunque, a posteriori, se suelan recordar (Frith, 2004). Hay que saber lo que hay que mirar. Es dificil saber qué despierta las primeras sospechas en la familia. Normalmente la preocupación se vuelve apremiante tras la acumulación de observaciones breves, cada una de ellas aparentemente insignificante.

"En mi opinión uno de los aspectos más crueles del autismo infantil es que, para los padres, es muy lento el proceso de darse cuenta de que a su hijo le pasa algo" (Werner, Dawson, Osterling, Dinno, 2000).

Si durante un largo tiempo no se les permite o ellos mismos se impiden ver lo evidente, va a resultar muy costoso superar esa ceguera, va a ser necesario que se les ayude a abrir los ojos y sobre todo a mirar de otro modo, encarando la situación.

\section{LA RECEPCIÓN DEL DIAGNÓSTICO}

La constatación por parte de los padres de que su hijo padece autismo, es muy dificil de asimilar. Generalmente no pueden creer lo que les dice el especialista, no es posible que su hijo padezca autismo, un trastorno grave, de origen orgánico, un trastorno hoy por hoy incurable y que requiere de tratamiento e intervención educativa lo antes posible y en un porcentaje alto de casos de por vida.

Hasta llegar a oír este diagnóstico, en muchas ocasiones, los padres han pasado por un verdadero calvario, un ir y venir de consulta en consulta, de especialista en especialista, desplazándose incluso de ciudad en ciudad, y por que no decirlo a veces también de país en país. La sospecha de que algo ocurre es confirmada por diversos profesionales, pero no existe el acuerdo respecto a lo que verdaderamente está ocurriendo.

Los padres suelen disponer de un repertorio amplio de historias, en muchos casos verdaderas historias de terror, sobre el tiempo que tardaron en diagnosticar a su hijo después de recurrir a la ayuda profesional. Es muy probable que hayan tenido que acudir a muchos especialistas hasta dar con la persona adecuada (Frith, 2004).

El peregrinaje en busca de un diagnóstico fiable, es sin duda una etapa dificil de superar, no sólo porque puede durar bastante tiempo, sino también por el enorme desgaste que genera en los padres. Por este motivo, es muy importante que los padres reciban un diagnóstico lo más pronto posible, ya que saber la verdad, por terrible que sea, es el principio de la aceptación de la realidad y es lo que abre el camino para tener el coraje de empezar a actuar. Recibir el diagnóstico es un alivio muy deseado, un extraño sentimiento cuando se reciben unas noticias tan devastadoras. Los padres sienten que su búsqueda ha terminado y que al menos pueden empezar a comprender y a darle sentido al mundo de su hijo. El diagnostico de TEA puede ser un alivio para las familias al saber por fin lo qué le ocurre a su hijo, pero generalmente suele ser un proceso doloroso, y el alivio suele ser usualmente corto.

El diagnóstico de autismo se basa en la conducta y la interpretación de la significación de una conducta alterada, ausente o retrasada depende de una sólida base de conocimiento clinico (Frith, 1994). Y dado que el autismo es un trastorno raro, hay relativamente pocos expertos que tengan experiencia de muchos casos. En la actualidad cada vez se va teniendo más claro que la elabora- 
ción de un diagnóstico de autismo es una tarea mutidisciplinar que requiere un nivel adecuado de especialización y dominio de los instrumentos y técnicas de evaluación y de apoyo a la persona y las familias.

Históricamente, el diagnóstico ha sido en nuestro país -y en otros muchos- un proceso extraordinariamente largo para las familias. Durante muchos años, y aún incluso hoy, es muy frecuente el retraso en el diagnóstico de autismo. Como el conocimiento del trastorno aumenta, está disminuyendo el tiempo que tarda en diagnosticarse y la edad a la que se hace. Sin embargo una evaluación que se basa en criterios conductuales no puede ser rápida. En los últimos años el tiempo que tardan las familias en obtener un diagnóstico desde que expresan sus preocupaciones al sistema de salud se han reducido ligeramente. Pero aún sigue existiendo un retraso diagnóstico muy considerable de aproximadamente 15 meses por término medio, siendo la demora mucho mayor en el caso del Trastorno de Asperger (Getea, 2005).

En la actualidad es factible llevar a cabo la localización de posibles casos de autismo en torno a los 18 meses al haberse identificado señales de alerta en el desarrollo (Baird, Charman, BaronCohen, 2000), pero no se trata de un diagnóstico sino de una detección temprana, un filtro que selecciona casos para una evaluación posterior. Los padres necesitan tiempo para familiarizarse con el proceso de evaluación, los motivos para llevarlo a cabo, lo que significan los resultados en su caso concreto y lo que puede hacerse en el presente y en el futuro (Frith, 2004). Una vez establecido el diagnóstico, los padres siguen necesitando ayuda y consejo.

\section{REACCIONES DE LOS PADRES}

Aceptar y comprender que un hijo tiene una discapacidad es un proceso que va más allá del mero conocimiento del hecho. Como cualquier suceso doloroso, la asimilación de esta situación dura un tiempo e incluso en algunos casos no llega a completarse nunca.

Si bien es cierto que la reacción de los padres ante el diagnóstico de autismo depende de variables como la experiencia previa, la demora en el diagnóstico, los signos presentes... podemos decir que en la mayoría de los casos el proceso de aceptación del hecho de tener un hijo con autismo se inscribe dentro de lo que se denomina respuesta a las crisis. Aunque existe un consenso importante con respecto a que esta respuesta pasa por varias y diferentes etapas, los sentimientos característicos de estas fases del proceso de duelo que viven los padres en ocasiones son ignorados por ellos mismos y en otras también por los profesionales. Es muy frecuente que experimenten un profunda tristeza, un sentimiento de pérdida o de ira (Harris, 2001).

Hasta la aceptación final del diagnóstico se vive algo muy parecido a las etapas de elaboración del duelo que Elisabeth Kübler-Ross ${ }^{1}$ (1989), estableció para explicar las reacciones ante la pérdida de un ser querido. De hecho, las etapas que atraviesan los padres hasta la aceptación de la discapacidad del hijo son muy similares a las del duelo. Siempre hay un sentimiento de pérdida al tener un hijo con una discapacidad: los

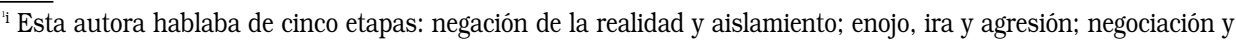
regateo; tristeza o depresión y aceptación.
} 
padres tienen que renunciar a la expectativa que todo padre tiene de tener un hijo sin ninguna dificultad.

En el caso de los trastornos del espectro autista tenemos que tener en cuenta que hasta llegar a ser diagnosticado pasa un tiempo en que los padres evidencian que "algo va mal" aunque no saben como se llama "ese mal". En este caso se trata de la pérdida de un hijo "idealizado", un hijo en el que se había puesto grandes ilusiones y esperanzas, una pérdida de planes y expectativas referente al futuro del niño y la familia. Se trata por tanto, de un proceso natural el que nieguen la evidencia del problema de su hijo. Como proceso requiere su tiempo y es preciso pasar por él. Los padres suelen atravesar por un proceso que implica hipersensibilidad, labilidad emocional, incertidumbre respecto a la situación familiar, sentimientos de culpabilidad, enojo, lo cual va a influir directamente en el trato con el hijo.

Cuando los padres reciben el diagnóstico inicial, cuando conocen la noticia de que su hijo tiene una discapacidad, entran en un estado de inmovilización, de shock, de bloqueo y aturdimiento general, debido a la grave e inesperada noticia. En el caso del autismo, como en otras muchas discapacidades, los padres no están preparados para escuchar que su hijo, padece un trastorno que no tiene cura. Es una fase de descreimiento y sorpresa, como ver el mundo desde fuera y que eso no les esté pasando a ellos. El efecto de esta fase sobre las familias sería leve si desde los primeros indicios se les apoyase y ayudase a identificar el trastorno.

Cuando se comunica que un hijo tiene un trastorno autista, es frecuente que los padres nieguen la evidencia, no quieran aceptar la realidad y busquen otras opiniones, otros diagnósticos. Intentan minimizar el problema, pensar que con el tiempo se pasará, que se trata sólo de un retraso madurativo. Este periodo es peligroso para las familias puesto que pueden terminar creyendo a las personas que les dan más esperanzas, sin analizar a fondo la base de sus argumentos y retrasando la búsqueda de intervenciones eficaces.

La irritación y la culpa también están presentes en los padres de niños con trastornos del espectro autista. Es una etapa en la que se repasa toda la vida del niño, incluso el embarazo intentando averiguar si pasó algo, si hubo alguna negligencia. Estos sentimientos de culpa se han visto favorecidos durante mucho tiempo -y aún hoy en algunos paísespor teorias que tendian a explicar que el autismo tenía un origen afectivo. Hoy en día, la confirmación avalada por la investigación científica sobre el origen orgánico del trastorno, no protege de este sentimiento de culpa, sino al contrario nos vemos rodeados por noticias 0 informes de profesionales que atribuyen el aumento de casos a una intoxicación por la administración de la vacuna triple vírica, a la ausencia de una dieta específica... hechos en absoluto constatados cientificamente pero que siguen generando sentimientos de irritación y culpa en las familias que lo único que quieren es saber lo que le ocurre a su hijo.

El inicio del convencimiento de que el hijo sufre una afectación grave e irreversible, -como es el autismo- conduce a los padres a un estado de profunda y lógica desesperanza, que puede derivar en un estado de depresión. Los padres pueden llegar a sentirse sobrecargados por el peso de estos sentimientos que les embargan y de los que casi nadie se atreve a hablar con claridad.

La fase de aceptación de la realidad se caracteriza por la superación de este 
estado de depresión y por la aplicación de medidas racionales para compensar el problema. En estos momentos los padres con un hijo con autismo, empiezan a sacar verdadero fruto del asesoramiento profesional y analizan las distintas opciones para proporcionar a su hijo la atención y tratamiento más adecuados. Adaptarse, reorganizarse y ayudar adecuadamente a los hijos no supone estar conforme con la discapacidad. Muchas familias aceptan a su hijo tal como es, pero toda la vida lamentan que sufra limitaciones y que tenga menos oportunidades de percepción, movilidad, comunicación o de autonomía. Pueden llegar a querer y disfrutar de sus hijos pero no pueden olvidar la discapacidad que presentan.

A estas fases pueden añadírseles según Rivière (1997a) las de comprobación, búsqueda de significado e interiorización real del problema causante de la crisis.

Las fases señaladas describen a grandes rasgos el proceso que viven los padres de un niño con discapacidad, pero el recorrido que realiza cada padre y cada madre es totalmente particular. Es importante entender que no todos los padres de niños con autismo pasan por todas las etapas, ni éstas tienen la misma duración. Pueden pasarlas con ritmos desiguales en la pareja o en el núcleo familiar. Asimismo tampoco tiene por qué ser un proceso continuo, pudiéndose dar pasos atrás y tardar mucho en la aceptación del diagnóstico o que uno de los padres no termine de aceptarlo en mucho tiempo, con la sobrecarga que ello supone para el otro progenitor. La duración de estas fases puede variar mucho de una familia a otra no solo en tiempo sino también en intensidad,... Son muchos los factores que van a influir en el transcurso del proceso, pero lo que está claro es que es preciso conocer las etapas y tenerlas en cuenta puesto que ignorar este camino va a influir en el resto de las acciones que llevan a cabo los profesionales.

Cuando se trabaja con una familia donde uno o varios miembros presenta un TEA, se puede observar cómo los padres vivencian el proceso de duelo de su hijo y aunque las reacciones que viven estos padres sean, según las investigaciones, normales, no lo son para algunos profesionales que desconocen este fenómeno y se preguntan innecesariamente la razón de porqué los padres no apoyan lo suficiente, su falta de implicación, la posición periférica del padre... e interpretan de manera inadecuada estas observaciones.

\section{CONVIVIENDO CON EL AUTISMO}

El hecho de que no todas las familias reaccionen y se adapten de la misma forma a la presencia de un niño con alteraciones en el desarrollo despertó en su momento el interés por el estudio de los factores que intervienen en esta variedad de respuestas (Freixa, 1993). La consideración de las familias de niños con discapacidad como "familias normales en circunstancias excepcionales" implica dejar de pensar en las familias en términos de carencias y problemas, para centrarse en sus necesidades. El modelo de las necesidades supone dar importancia a la respuesta social y en concreto a la adecuación de los servicios a sus necesidades. Se abandona la idea de que son las características del niño el único factor determinante de las respuestas adaptativas que dan las familias. Hay que tener en cuenta también otros factores como las creencias y estilos de comportamiento desarrollados por los padres, la calidad de las relaciones familiares o de los sistemas de apoyo externo (Simón, Correa, Rodrigo, y Rodríguez, 1998). 
Desde perspectivas globales y sistémicas se identifican algunos elementos entre las características del niño, de la familia y de los recursos del entorno, que claramente aumentan el riesgo en la familia, sobre todo si se dan de forma combinada.

Una variable especial a tener en cuenta a la hora de trabajar con familias con hijos con discapacidad es la capacidad de los padres para afrontar situaciones de estrés. Los distintos estilos de los padres para afrontar el estrés explican el hecho de que familias en situaciones aparentemente muy similares (misma discapacidad y edad de los hijos, similar situación sociofamiliar, acceso a los mismos recursos) muestren a veces grados de adaptación extremadamente diferentes.

Si la alteración de cualquier miembro de una familia afecta al conjunto, la realidad de un niño con trastorno del espectro autista trastoca todos los niveles de responsabilidad, madurez, dedicación y recursos de los que dispone la familia así como sus relaciones con otras familias y amigos. El carácter generalizado de las alteraciones de las personas con trastornos del espectro autista y su permanencia a lo largo de la vida hace que las implicaciones que tiene para las familias el nacimiento y convivencia con un hijo/a con autismo abarquen todos los ámbitos y generen necesidades en relación a todos los tipos de servicios (Belinchón, 2001).

Distintos estudios (Oizumi, 1997; Sanders y Morgan, 1997; Karisi y Sigman, 1997; Maan, 1996; Wolf, Noh, Fisman y Speechley, 1989) demuestran que la convivencia con un hijo con TEA pro- voca en los padres niveles de estrés muy superiores a los producidos por la paternidad en sí misma e incluso a la paternidad de hijos con otras discapacidades. Los patrones de depresión reactiva y aumento de estrés (Moes, 1995) que presentan los padres de niños con TEA se relacionan con numerosos factores: la dificultad para comprender qué le sucede al niño, la naturaleza inherentemente culpógena del propio autismo, la gran dependencia mutua que se crea entre los niños con autismo y sus padres, la dificultad para afrontar las alteraciones de conducta y el aislamiento, la falta de profesionales expertos, la limitación de las oportunidades vitales y de relación que supone en un primer momento la existencia de una atención constante al niño, etc.

"La situación de depresión y estrés puede aumentar como si fuera una lupa, pequeños problemas familiares, convirtiéndolos en dificultades serias de relación en la pareja. Complicarse, tomando la forma de un bucle de culpabilizaciones reciprocas. Dificultar la atención a los hermanos. Tener, en fin, consecuencias negativas de diversos órdenes en la economía de las relaciones familiares” (Rivière, 1997a, p. 39).

Desde que se iniciaron los primeros estudios sobre el efecto que provocaba el autismo en la familia (DeMyer, 1979) se ha tratado de analizar la influencia que ciertos factores tienen sobre el estrés familiar $^{2}$ en el caso del autismo. Uno de los modelos que más se ha usado en esta búsqueda de factores es el modelo multivariado de factores en interacción (modelo simple ABCX) ideado por Hill (1949, 1958) y utilizado para determinar el estrés en familias con distintos trastor-

\footnotetext{
${ }^{\text {iii }}$ El estrés familiar se define como un estado que surge por un desequilibrio entre la percepción de las demandas y las capacidades para hacerles frente.
} 
nos. En dicho modelo, el factor "a" (evento estresor) interactuando con "b" (recursos) y con "c" (la definición que la familia hace del evento) produce " $x$ " (crisis).

Este modelo no explicaba los ajustes que hace la familia para adaptarse a las nuevas demandas que se van planteando a lo largo del tiempo. Para solventar esta carencia McCubbin y Patterson (1983) amplian el modelo simple añadiendo nuevas variables. Proponen el Modelo del Doble ABCX de Ajuste y Adaptación en el que el factor aA (evento estresor y/o acumulación de demandas) en interacción con el factor bB (recursos existentes antes de la crisis y nuevos recursos) y con el factor cC (percepción o significado que la familia atribuye al acontecimiento estresante y a su capacidad para manejarlo) produce un resultado de adaptación o factor $\mathrm{xX}$ (niveles de estrés) (Pozo, Sarriá y Méndez, 2006).

Bristol (1987) ha sido uno de los autores pioneros que ha utilizado este modelo en el caso del autismo, evaluando como factor aA las características del niño, como factor bB los apoyos (formal e informal con el que cuenta la familia) y como factor $\mathrm{cC}$ la influencia que una definición negativa del problema tiene en la adaptación familiar.

Siguiendo a Pozo, Sarriá y Méndez (2006) podemos afirmar que el resultado final de adaptación, no sólo depende de las características de la persona con autismo, sino que se trata de un proceso más complejo, donde la percepción del problema y los apoyos intervienen de manera significativa.

El impacto de la discapacidad depende en gran medida de los recursos con los que cuente la familia y de la percepción que la propia familia tenga de la situación (Bristol, 1987) de manera que el apoyo familiar y el nivel de satisfac- ción marital amortigua mucho los efectos del estrés.

Para favorecer la convivencia con un hijo con TEA es necesario conocer en profundidad las caracteristicas de dichos trastornos y aceptar las importantes limitaciones que implican a nivel de las relaciones sociales, la comunicación y la imaginación. La vida con una persona con autismo es muy dura, a las limitaciones propias del autismo hay que sumarle en muchos casos los problemas de comportamiento que de por sí son muy dificiles de tratar, la hiperactividad, las alteraciones del sueño y de la alimentación. Es una convivencia que desgasta, que genera gran frustración, como por ejemplo observar al hijo sumido gran parte del tiempo mirándose las manos 0 jugando con una rueda... e intentar implicarle en otras actividades y no recibir respuesta por su parte.

\section{AFECTACIÓN A LA DINÁMICA FAMILIAR}

Cualquier patología de uno de los miembros del grupo familiar, como es el autismo en un hijo, modifica no sólo el contexto y el clima familiar, sino también a las personas que integran la familia y a las relaciones existentes entre ellas (Polaino-Lorente, 1997). Las interrelaciones y el ambiente de la familia se ve alterado de tal modo que es necesario que todos los miembros intenten adaptarse a la nueva situación de forma que la familia siga disfrutando de un ambiente sano, cálido y sereno (Doménech, 1997).

La persona con autismo desde muy pequeña no puede interactuar normalmente con sus padres y estos no pueden, por mucho que lo deseen establecer con ellos una relación normal. Los comportamientos cotidianos de interacción entre un niño y una madre, tales como el con- 
tacto ocular, la mirada, la sonrisa, los gestos, las posturas, el acercamiento, el abrazo, etc, no ocurre entre un bebé con autismo y su madre. Este hecho tan importante tiene sus consecuencias en las primeras interacciones que la madre establece con su bebé, pero también en los sentimientos de la madre que no va a ver realizado su rol de madre, pudiendo afectar a la relación con su pareja y con el resto de los hijos.

El tener un hijo o hija con autismo normalmente produce un impacto que afecta enormemente a la vida familiar, pudiendo distorsionar el ambiente familiar. Los padres de un hijo con autismo van a tener que afrontar graves dificultades. El autismo causa frustración, estrés, confusión en la familia. Puede producir en los padres problemas emocionales especiales, ya que se diagnostica transcurridos los primeros años de vida, el desarrollo físico del niño no presenta ningún motivo de alarma y a veces incluso tiene tales habilidades que no parece que tenga un problema (Wing, 1998).

No es lo mismo saber que un hijo está enfermo que convivir con él cada dia, luchando continuamente por lo relativo a sus alteraciones del lenguaje, su comportamiento agresivo, su carencia de habilidades sociales, su incomunicación, estereotipias y conductas autolesivas y destructivas, etc., en una palabra por esa interacción que es preciso establecer con él y que aparece quebrada (PolainoLorente, 1997).

A todo esto deben añadirse otras muchas circunstancias que ahondan todavía más el sufrimiento e incomprensión de los padres, y en especial de la madre, como son la pérdida de expectativas de control sobre la conducta del hijo, las atribuciones negativas que sobre él hace, el miedo al futuro, la renuncia a su trabajo, las cargas económicas adicionales que ello supone, la escasez de recursos económicos, etc.

El riesgo de estrés en estas familias, tal y como ya se ha comentado, es muy alto y con ello, el riesgo de vivir en un clima constante de tensión y angustia. El estrés ejerce un efecto negativo sobre el clima emocional, las relaciones de la familia con amigos, las actividades de ocio, la organización económica familiar, la actividad y dedicación de la madre fuera del hogar, las interacciones dentro del núcleo familiar y, en general, el equilibro psíquico y físico de los adultos de la casa (Bebko, Konstantareas y Springer, 1987).

De manera resumida podemos decir que la dinámica familiar se puede ver afectada por varios factores: la autoatribución y atribución de tareas y roles, entre el padre y la madre, en cuanto al cuidado del hijo con autismo; el grado de sensibilidad (resistencia) de cada uno de los miembros de la pareja hacia los problemas que presenta su hijo; el apoyo recibido por parte del cónyuge; la satisfacción conyugal y la felicidad personal de cada uno de ellos; la percepción que los padres tienen de los diversos trastornos asociados al autismo y su valoración sobre el nivel de estrés que cada uno conlleva; el entorno social.

Tener unas expectativas realistas con respecto al hijo con autismo y mantener un clima familiar de calidad son dos aspectos fundamentales en la convivencia positiva (Doménech, 1997) y que previenen de posibles complicaciones a nivel personal, familiar y social.

Si bien es cierto que la calidad de la comunicación familiar varía con el tiempo y con las etapas evolutivas de la vida, es preciso que los padres y los hijos no pierdan el contacto mutuo con los aspectos importantes de sus vidas. Los secre- 
tos familiares, los significados implícitos y los silencios son los mayores enemigos para el desarrollo de una buena comunicación familiar (Harris, 2001). Cuando una persona con autismo llega a un sistema familiar la comunicación está amenazada y vulnerable por la necesidad de ocultar el dolor, la incertidumbre, la rabia y las preocupaciones con el fin de mantener la homeostasis familiar.

Los hermanos comparten las crisis de la vida y los ritos de transición esenciales para la identidad cultural y social, por esto las relaciones fraternas tienen una gran trascendencia psicológica y evolutiva.

La calidad global de la vida familiar tiene una influencia importante en la forma de relacionarse los hermanos. Es más probable que los padres que formen una buena pareja y resuelvan sus diferencias, tengan hijos que hagan lo mismo. Del mismo modo es fácil que los hijos de padres que los atiendan bien mantengan unas buenas relaciones (Boer, Goedhart y Treffers, 1992). Para ser un padre o una madre que cumpla bien sus funciones hace falta una serie de cualidades como transmitir respeto, cariño y amor, ser coherente con respecto a las expectativas y reglas, establecer limites claros ante las conductas inadecuadas de los niños y facilitar unas rutinas infantiles previsibles pero flexibles. En las familias en las que haya problemas graves entre los padres, los hermanos pueden ser una fuente de apoyo mutuo importante (Jenkins, 1992), pero estas relaciones no pueden reemplazar el amor y el saber de un progenitor competente. Todo esto pone de manifiesto la gran importancia de que los padres mantengan un matrimonio sano, no sólo para ellos mismos sino también para sus hijos. La interacción entre los hermanos es extremadamente sensible a la calidad de la interacción entre los padres.
"Con frecuencia nos centramos tanto en las intensas necesidades educativas del niño con autismo que tendemos a pasar por alto las necesidades de los demás hermanos. Sin embargo cuando escucho sus voces en los grupos de apoyo a las familias o en entrevistas individuales me sorprende la urgencia de sus necesidades" (Harris, 2001, p. 7).

Por otra parte es importante destacar que las familias que tienen hijos con dificultades similares se convierten en fuente insustituible de apoyo emocional e información. La vía para establecer nuevos contactos son las asociaciones de padres de personas con discapacidades. Inicialmente muchas familias no buscan espontáneamente esta vía de asociación, con frecuencia la pertenencia a una determinada asociación se inicia por la posibilidad de recibir un servicio o por la recomendación de los profesionales que atienden a la familia. No obstante es importante tener en cuenta que el contacto con otros padres que viven situaciones similares debe contar con algunas garantias, ya que no todas las familias están en condiciones de servir de apoyo efectivo a otros padres (Paniagua, 1999).

"Lo que si compartí con las madres de hijos deficientes es la soledad, acrecentada si tu hijo está gravemente afectado. La carencia de alguien con quien compartir tu dura realidad y al experimentar que tu hijo también está solo" (Ródenas de la Rocha, 2002, p. 35).

\section{AJUSTES QUE HACE LA FAMILIA PARA ADAPTARSE A LAS NUEVAS DEMANDAS QUE SE VAN PLANTEANDO A LO LARGO DEL TIEMPO}

La convivencia en el hogar de un niño con autismo suele ser dificil, las familias se ven sometidas desde el principio 
a modificaciones severas de su régimen de vida habitual con limitaciones exageradas de su independencia. Las adaptaciones físicas de las viviendas, la disminución del tiempo libre, las restricciones de la vida social, la incertidumbre frente a un futuro que se prevé oscuro y al mismo tiempo incierto, la polarización de la vida familiar alrededor del miembro con autismo modifican tanto la vida de la familia que se requiere llevar a cabo ajustes en la vida familiar que permitan una vida lo más armónica posible.

Ante un problema tan complejo como es el autismo, los conocimientos generales y el sentido común de los padres que, normalmente son suficientes para criar y educar a la mayor parte de los niños, no lo son en este caso. Los padres tienen que aprender a vivir con un hijo con unas características especiales que exige unas adaptaciones importantes en todos los sentidos. La vivienda tiene que reorganizarse al igual que cada una de las personas que convivan en el hogar familiar.

Para hacer frente al estrés que supone tener un hijo con autismo y mantener un clima familiar aceptable, cada familia dispone de unos recursos que pueden ser muy distintos en cada caso. Estos recursos pueden referirse a las características de personalidad, motivaciones y valores de los componentes de la familia y de la forma de relacionarse entre ellos; de la unidad familiar y de la atmósfera que reina en el hogar; y/o también de los apoyos externos que proporcione la comunidad (Doménech, 1997).

Los sistemas de valores y creencias de la familia son los factores más importantes que influyen en la adaptación y en la capacidad de resistencia y flexibilidad de las familias. Estos sistemas comprenden la visión que se tiene sobre el mundo, los valores y las prioridades. Las visiones sobre el mundo consisten en las opiniones de la familia sobre el ambiente social y cultural y sobre el sitio que ocupa esa familia en el mundo. Los valores familiares se refieren a los principios fundamentalmente operativos por los que la gente dirige sus vidas y organiza las vidas de sus familias. Las prioridades de la familia tienen que ver con las actividades y roles que consideran más importantes (King, Zwaigenbaum, King, Baxter, Rosenbaum, Bates, 2006).

En muchas ocasiones la familia debe optar por modificar fisicamente la vivienda para poder hacer frente a los efectos de los problemas de conducta que presenta la persona con autismo.

También es preciso modificar la forma de comunicación. Las personas con TEA tienen un lenguaje particular, miran el mundo desde una perspectiva singular y se perciben a sí mismos y a los demás de manera diferente a como nos percibimos el resto de las personas. Viven en un mundo de percepción directa e inmediatez, ven el mundo sin metáforas. Los padres necesitan disponer de ayudas que les permitan entender este lenguaje enigmático y contradictorio en el que se expresa su hijo. No utilizan el lenguaje de manera cotidiana para negociar el mundo social, su lenguaje a menudo se limita a tareas cotidianas y a peticiones sencillas que satisfacen sus propias necesidades. Si no hablan no sustituyen los significados no verbales de la comunicación. Esto hace que los padres de niños/as con autismo a menudo tienen que adivinar cuál puede ser el significado de un comportamiento (Szatmori, 2006). En los casos en los que hay lenguaje, en muy contadas ocasiones la conversación que mantienen es recíproca, normalmente hablan sin parar de sus temas de preferencia. 
Una vez que los padres son capaces de entender a su hijo, de saber qué es lo que le hace pensar y sentir de manera diferente, la vida les resulta mucho más llevadera. Aprender a mirar el mundo a partir de la mente de su hijo/a, influye no solo en los padres sino en toda la familia, incluyendo el hijo con autismo. Entender el modo en el que el niño piensa y siente, y de qué modo eso se traduce muy a menudo en un comportamiento desconcertante, a veces inquietante, permite superar muchos de los problemas que impiden que las relaciones entre padres e hijos sean gratificantes y las intervenciones efectivas.

Los ajustes temporales son de gran importancia. Un aspecto a destacar es el aumento de dedicación que en general supone un hijo con necesidades especiales El tiempo libre disminuye lo que lleva a una restricción considerable de la vida social. Requieren muchos más cuidados físicos, así como más tiempo de interacción y más situaciones de juego o estudio compartido. El desarrollo de programas de estimulación, actividades de ocio y refuerzo familiar, revisiones médicas, orientación psicopedagógica, búsqueda de opciones y recursos... suponen para los padres un esfuerzo personal que es preciso tener en cuenta (Paniagua, 1999). Por otra parte muchos padres, pero sobre todo madres, sacrifican durante años sus posibilidades de ocio y desarrollo profesional por la dedicación al hijo.

Los padres y hermanos deben ajustar sus percepciones, actitudes, creencias, ideas, ... aprendiendo a interpretar el comportamiento de la persona con TEA y llegando a aceptar que las personas con autismo son personas con un modo diferente de pensar y de procesar la información.

Sin comprender a la persona con autismo los padres no se pueden relacio- nar con ella. Es preciso que los padres comprendan el trastorno, que conozcan los sintomas que afectan a todos los aspectos del comportamiento y cómo esto se manifiesta en la vida cotidiana. Este conocimiento ayuda a restablecer un sentido de relación entre los padres y el hijo.

Para conseguir esta comprensión los padres pueden observar a su hijo/a, imaginar las sensaciones que le producen los estímulos teniendo en cuenta su especial sensibilidad a los mismos, mirar con detenimiento los detalles que tanto le llaman la atención..

Los logros de una persona con TEA parecen decepcionantes sólo si se miran desde fuera, cuando son medidos por el rasero de los demás. Es mucho mejor considerar los resultados desde el punto de vista de la persona con TEA, en relación con los obstáculos que ha tenido que superar para llegar hasta donde ha llegado. Los triunfos de una persona con TEA a menudo son privados, los padres no pueden dar nada por supuesto, cada paso, cada avance es vivido como una victoria y sobresale en el flujo diario de acontecimientos cotidianos "como un destello de luz brillante” (Szatmari, 2006, p. 156).

\section{A MODO DE REFLEXIÓN}

Las personas afectadas de autismo constituyen, dentro de los tipos de discapacidad, uno de los grupos de menor autonomía, y en el que las familias sufren un desgaste más importante desde los primeros años de la vida del hijo y durante toda su vida. Esto se debe a que este sindrome, como ya se ha comentado en varias ocasiones, conlleva además de un déficit cognitivo global presente en la mayor parte de los afectados, trastornos graves de las relaciones 
interpersonales y la comunicación y problemas severos de conducta, con lo que su autonomía personal se ve, en todo momento muy afectada.

El apoyo y orientación de profesionales en los primeros momentos son primordiales para que los padres puedan aprender a comprender y aceptar a su hijo/a. Una vez que logran mirar el mundo a través de los ojos del niño con TEA es posible comprender en gran medida sus comportamientos, incluso los problemáticos y desconcertantes. Esta manera de ver las cosas lleva a los padres -y demás personas cercanas a la persona con TEA- a enfocar mejor y de una manera más respetuosa el tratamiento y a obtener un mejor resultado en el futuro. Tener una familia o un educador que comprenda qué sucede dentro de la mente de un niño o un adulto con TEA es de gran importancia porque al comprender se establece una especie de empatía con él y esa empatía lleva a desarrollar una relación especial en ausencia de la cual cualquier programa de intervención está destinado a fracasar.

Al no tener un nombre con el que designar el desconcertante comportamiento de sus hijos, los padres tienen miedo de lo desconocido y miran hacia el futuro con aprehensión y pavor. Pero el hecho de comprender y conocer las implicaciones que conlleva el autismo supone un gran paso a la hora de aceptar la desesperación que muchas familias sienten.

Los padres tienen que enseñar a los maestros, educadores, cuidadores..., el modo de interpretar la mente de su hijo, de este modo se posibilita la comprensión y se puede hacer que la incorporación a lo distintos recursos existentes (centro educativo, centro de día, vivienda...) sea más positiva. A su vez los distintos profesionales (educación, sanidad, servicios sociales) deben ser sensibles y receptivos a estas enseñanzas, dispuestos a cambiar su percepción de la persona con autismo y entregados a mejorar su calidad de vida.

"No es el mundo un lugar mejor con las personas con autismo? ¿Por qué perder esto? La gente con autismo es especial, son únicos. Y con la ayuda y el apoyo correctos ellos también pueden hacer algo junto con el resto de la sociedad" (Congreso Autismo Europa, 2000, en prólogo de Ródenas de la Rocha, 2002). 


\section{BIBLIOGRAFÍA}

Baird, G., Charman, T. y Baron-Cohen, S. (2000). A screening instrument for autism at 18 months of age : A 6-year follow-up study, Journal of de American Academy of Child and Adolescent Psychiatry, 39, 649-702.

Bebko, J., Konstantareas, M. y Springer, J. (1987). Parent and professional evaluations of family stress associated with characteristics of autism. Journal of Autism and Developmental Disorders, 17 (4), 565-577.

Belinchón, M. (Dir.) (2001). Situación y necesidades de las personas con trastornos del espectro autista en la Comunidad de Madrid. Madrid: Caja Madrid.

Boer, F.; Goedhart, a.w. y Treffers, P.D. (1992). Siglings and their parents. En F. Boer y J. Dunn (Eds.) Children's sibling relationships. Developmental and clinical issues (pp. 41-54). Hillsdale:Erlbaum Associates.

Bristol, M. (1987). Mothers of children with autism or communication disorders: successfull adaptation and the double ABCX Model. Journal of Autism and Developmental Disorders, 17 (4), 469486.

Cuxart, F. (1997). El impacto del niño autista en la familia. En En A. Polaino-Lorente, E. Doménech y F. Cuxart, El impacto del niño autista en la familia (61-71). Navarra: Rialp.

DeMyer, M.K. (1979). Parentes and children in autism. New York: Wiley.

Doménech, E. (1997). El estrés de los padres y el clima familiar del niño autista. En A. PolainoLorente, E. Doménech y F. Cuxart, (eds.) El impacto del niño autista en la familia (95-110). Navarra: Rialp.

Freisa, M. (1993). Familia y deficiencia mental. Salamanca: Amarú.

Frith, U. (1994). Autismo. Madrid: Alianza (Orig. 1989).

Frith, U. (2004). Autismo. Hacia una explicación del enigma. Madrid: Alianza. (Orig. 2003).

García Villamisar, D.; Cuxart, F. y Jiménez Casas, C. L.(2006). Las personas con autismo ante la futura Ley de Promoción de la Autonomía Perso- nal. Revista del Ministerio de Trabajo y Asuntos Sociales, 60, 113-127.

Getea (Grupo de Estudio de trastornos del Espectro Autista) (2005). Instituto de Salud Carlos III. http//iier.isciii.es

Jenkins, J. (1992). Sibling relationships in disharmonious homes: Potencial difficulties and protective effects. En En F. Boer y J. Dunn (Eds.) Children's sibling relationships. Developmental and clinical issues (pp. ¿??). Hillsdale:Erlbaum Associates.

Harris, S. L. (2001). Los hermanos de niños con autismo. Su rol específico en las relaciones familiares. Madrid: Narcea.

Hill, R. (1949). Families under stress: adjustment to the crisis of war, separation and reunion. New York: Basic Books.

Hill, R. (1958). Generic features of families under stress. Social Casework, 49, 139-150.

Karisi, C. y Sigman, M. (1997). Linking parental perceptions to interactions in young children with autism. Journal of Autism and Developmental Disorders, 27 (1) 39-57.

King, G.A.; Zwaigenbaum, L.; King, S.; Baxter, D.; Rosenbaum, P. y Bates, A. (2006). Cambios en los sistemas de convicciones/valores de las familias de niños con autismo y sindrome de Down, Revista Síndrome de Down 23, 51-58.

Kübler-Ross, E. (1989). La muerte y los moribundos. Barcelona: Luciérnaga (Original 1969).

Maan,C. (1996). Stress, coping and adaptation in families with young autistic children. Dissertation Abstracts Internacional. The sciencies and engineering, 56.

McCubbin, H. I. y Patterson, J. M. (1983). The family stress process: the double ABCX model of adjustment and adaptation. In H. I. McCubbin, M. B. Sussman y J. M. Patterson (eds.) Social stress and he family (7-37). New York: Haworth.

Moes, D. (1995). Parent education and parenting stress. In Koegel, R.L y Koegel, L. K (eds.) Teaching children with autism: Strategies for initiating positive interactions and improving learning opportunities (79-94). Baltimore: Brookes. 
Oizumi, J.J. (1997). Assesssing maternal functioning in familias of children with autism. Dissertation Abstracts Internacional. The sciencies and engineering, 57.

Paniagua, G. (1999). Las familias de niños con necesidades educativas especiales. En A. Marchesi, C. Coll y J. Palacios (comp.) Desarrollo psicológico y educación. 3 Trastornos del desarrollo y necesidades educativa especiales (469-493). Madrid: Alianza.

Polaino-Lorente, A. (1997). El estrés de los padres del niño autista: ¿Es el mismo para el padre que para la madre? En A. Polaino-Lorente, E. Doménech y F. Cuxart, El impacto del niño autista en la familia (83-93). Navarra: Rialp.

Pozo, P.; Sarriá, E. y Méndez, L. (2006). Estrés en madres de personas con trastornos del espectro autista. Psicothema, 18 (3), 342-347.

Rivière, A. (1997a). Definición, etiología, educación, familia papel psicopedagógico en el autismo. Curso de desarrollo normal y autismo. Santa Cruz de Tenerife.

Rivière, A. (1997b). El tratamiento del autismo como trastorno del desarrollo: Principios generales. En A. Rivière y J. Martos (comp.) El tratamiento de autismo. Nuevas perspectivas (23-59). Madrid: Ministerio de trabajo y Asuntos Sociales.

Ródenas de la Rocha, C. (2002). Pablo... Autis ta. Experiencias de una madre y su hijo con retraso mental y autismo. Sevilla: Fundación Verbum.
Sanders, J. L. y Morgan, S. B. (1997). Family stress and adjustment as perceived by parents of children with autism or Dawn Sindrome: implications for the intervention. Child and family behaviour therapy, 19(4), 15-32.

Simón, M. I.; Correa, N.; Rodrigo, M. J. y Rodríguez, M. A. (1998). Desarrollo y educación familiar en niños con cursos evolutivos diferentes. En M. J. Rodrigo y J. Palacios (coords) Familia y desarrollo humano (pp. 445- 464). Madrid: Alianza.

Stanton, M. (2002). Convivir con el autismo. Una orientación para padres y profesores. Barcelona: Paidós (Orig. 2000).

Szatmari, P. (2006). Una mente diferente. Comprender a los niños con autismo y sindrome de Asperger. Barcelona: Paidós (Original 2004).

Werner, E., Dawso, G., Osterling, J. y Dinno, N. (2000). Brief report: Recognition of autism spectrum disorder before one year of age: A retrospective study based on home videotapes. Journal of Autism and Developmental Disorders, 30, 157-162.

Wing, L. (1998). El autismo en niños y adultos. Barcelona: Paidós.

Wolf, L. C.; Noh, S.; Fisman, S. N. y Speechley, M. (1989). Brief report: Psychological effects of parenting stress on parents of autistic children. Journal of Autism and Developmental Disorders, 19 (1), 157-166. 